\title{
The emergence of a new science of the mind: immunology benefits the mind
}

Molecular Psychiatry (2010) 15, 337-338;

doi:10.1038/mp.2010.22

A new era is emerging in the understanding of the cross-talk between the immune system and the brain, based on an appreciation of the role of circulating immune cells in supporting brain plasticity, as manifested in normal behavior, cognition, and mental activity. This novel aspect of the body-mind duality was denied for decades because of the general perception of the brain as a tissue behind walls, and because of the assumption that the brain needs to be protected from immune cells in order to function optimally. The recent appreciation of the supportive role of immunity in behavior and cognition in both health and disease is expected to directly impact research in Neuroimmunology and Psychoneuroimmunology, creating a new discipline, Immunology of the Mind.

The concept of 'mind' in its modern sense, encompassing cognition and mental activity, on one hand, and the fields of immunology, neuroimmunology and psycho-neuroimmunology, on the other hand, has gone through dramatic conceptual changes over the last decades. Thus, for example, the notion of mind has been expanded from a philosophical and psychological concept to a physical existence, and, consequently, is an area that is heavily investigated by neuroscientists. The general perception of the role of the immune system has been expanded to include not only host defense but also somatic maintenance and tissue repair. Likewise, the fields of neuroimmunology and psychoneuroimmunology have evolved from disciplines that view the cross-talk between the peripheral immune system and the brain either in terms of how the mind affects the immune system ${ }^{1}$ or in terms of how immune system pathologies (such as inflammation) disrupt the mind, ${ }^{2,3}$ to fields that appreciate the pivotal role of circulating immune cells in helping sustain the healthy brain. ${ }^{4-7}$ Thus, for example, it has been commonly accepted that mental stress (depending on its severity) either harnesses immune cells to support the body's function (excluding that of the brain) or suppresses healing; ${ }^{8}$ however, the possibility that circulating immune cells may actually protect the brain's function from the consequences of stress was only recently recognized. ${ }^{9,10}$ (Cover page) The novel dimensions that were identified in the cross-talk between the brain and the immune system are focused on understanding how the peripheral immune system contributes to brain plasticity, and in cases of brain pathologies, to reveal how immune insufficiency deprives the brain of necessary assistance required for normal behavior, cognition, mental activity and repair. ${ }^{11-15}$ This transition in understanding the immune system-brain interface has a direct impact on the directions of research attempting to reveal the mystery of brain diseases and aging. Accordingly, even congenital neuropsychological diseases with delayed onset (for example, autism, schizophrenia, and Tourette's syndrome) may be viewed not necessarily as pathologies caused by autoimmune components attacking the brain, but as a potential reflection of a specific type of immune deficiency.

In fact, the ability of the immune system to support the function of the healthy mind is a novel aspect of the body-mind dialogue. Such support can be viewed as an outcome of the contribution of circulating immune cells to restoring homeostasis following any positive or negative stimulus, and may be manifested in cognitive ability, or in coping with stress. Similar immune support might also explain the link between nutrition or physical exercise and cognitive ability.

Homeostatic mechanisms allow the individual to 'bounce back', or recover from physiologically or psychologically stressful situations, and to return to a 'set point' following internal or external deviation. In the brain, several intrinsic levels of homeostatic mechanisms exist to maintain dynamic neuronal circuits despite fluctuations in their inputs; these range from the maintenance of ionic balance and synaptic plasticity, to structural changes and neurogenesis. The involvement of the immune system as an integral part of these physiological processes of restoration (Cover Page) also calls for revisiting the definition of immune privilege and establishing new principles of 'Immunology of the Mind'.

Importantly, this new dimension to understanding the factors involved in the brain's homeostasis identifies a possible common factor or mechanism linking mental disease and neuropsychological disorders (for example, depression, post-traumatic stress disorder, schizophrenia, Tourette's syndrome, autism, attention and eating disorders), and other neurodegenerative diseases (Alzheimer's disease), and agerelated dementia, and portends rapid progress in developing treatments for hitherto incurable brain disorders.

This special issue, devoted to the Immunology of the Mind, introduces a new discipline at the interface of Immunology, Neurosciences, Psychology, Neurology, Molecular Psychiatry and Behavioral Neurosciences, 
$338 \quad$ and is intended to establish the role of peripheral immunity as an integral part of the normal physiology of the brain, and consequently, to explain brain pathology as a deficit of such immune activity.

The solicited Feature Review will introduce the emerging principles of 'Immunology of the Mind', and their implications for normal mental function and dysfunction. The selected original papers that are included here were not solicited, but were selected from papers that were accepted for publication in Molecular Psychiatry and are relevant to this topic.

\section{Acknowledgments}

MS is supported in part by ERC Advanced Research Award.
M Schwartz

Department of Neurobiology, The Weizmann Institute of Science, Rehovot, Israel

E-mail: Michal.schwartz@weizmann.ac.il

\section{References}

1 McEwen BS. N Engl J Med 1998; 338: 171-179.

2 Enstrom AM et al. Curr Opin Investig Drugs 2009; 10: 463-473.

3 Goldsmith CA, Rogers DP. Pharmacotherapy 2008; 28: 730-741.

4 Kipnis J et al. Proc Natl Acad Sci USA 2004; 101: 8180-8185.

5 Ziv Y et al. Nat Neurosci 2006; 9: 268-275.

6 Ron-Harel N, Schwartz M. Trends Neurosci 2009; 32: 367-375.

7 Wolf SA et al. J Immunol 2009; 182: 3979-3984.

8 Maestroni G. Nat Immunol 2004; 5: 763.

9 Cohen $\mathrm{H}$ et al. J Neurobiol 2006; 66: 552-563.

10 Lewitus GM et al. Brain Behav Immun 2008; 22: 1108-1114.

11 Kipnis J et al. Trends Immunol 2008; 29: 455-463.

12 Moalem G et al. Nat Med 1999; 5: 49-55.

13 Lewitus GM, Schwartz M. Mol Psychiatry 2009; 14: 532-536.

14 Lewitus GM et al. Biol Psychiatry 2008; 65: 283-288.

15 Miller AH. Brain Behav Immun 2010; 24: 1-8. 